\title{
Prognostic impact of expression and methylation status of DENN/MADD domain-containing protein 2D in gastric cancer
}

\author{
Mitsuro Kanda • Dai Shimizu $\cdot$ Shuji Nomoto $\cdot$ Hideki Takami $\cdot$ Soki Hibino $\cdot$ Hisaharu Oya $\cdot$ Ryoji Hashimoto $\cdot$ \\ Masaya Suenaga Y Yoshikuni Inokawa Daisuke Kobayashi $\cdot$ Chie Tanaka Suguru Yamada $\cdot$ Tsutomu Fujii $\cdot$ \\ Goro Nakayama $\cdot$ Hiroyuki Sugimoto $\cdot$ Masahiko Koike $\cdot$ Michitaka Fujiwara $\cdot$ Yasuhiro Kodera
}

Received: 9 January 2014/ Accepted: 12 March 2014/Published online: 3 April 2014

(c) The International Gastric Cancer Association and The Japanese Gastric Cancer Association 2014

\begin{abstract}
Background Patients with advanced gastric cancer (GC) have an adverse prognosis even after curative resection. Development of novel diagnostic and therapeutic approaches for GC is urgently required.

Methods The expression and methylation status of DENN/MADD domain-containing protein 2D (DEN$N D 2 D$ ), a member of the membrane trafficking proteins, were evaluated in $12 \mathrm{GC}$ cell lines and 112 pairs of surgical specimens. Subgroup analysis based on tumor differentiation, location, and morphology was also performed. Expression and distribution of DENND2D protein were determined by immunohistochemistry.

Results The majority of GC cell lines (75\%) and tissues (79 \%) showed reduced expression of DENND2D mRNA compared with noncancerous gastric tissues. GC tissues showed a significantly lower mean expression level of mRNA and a higher frequency of promoter hypermethylation of DENND2D than corresponding noncancerous tissues. No significant differences in DENND2D mRNA expression and methylation status were found between GC subtypes categorized by tumor differentiation, location, and morphology. The expression patterns of DENND2D protein were confirmed to be consistent with those of DENND2D mRNA. Downregulation of DENND2D mRNA in GC tissues was significantly associated with factors
\end{abstract}

M. Kanda $(\bowtie) \cdot$ D. Shimizu $\cdot$ S. Nomoto $\cdot$ H. Takami ·

S. Hibino $\cdot$ H. Oya $\cdot$ R. Hashimoto $\cdot$ M. Suenaga ·

Y. Inokawa - D. Kobayashi - C. Tanaka - S. Yamada .

T. Fujii · G. Nakayama · H. Sugimoto · M. Koike ·

M. Fujiwara $\cdot$ Y. Kodera

Department of Gastroenterological Surgery (Surgery II), Nagoya

University Graduate School of Medicine, 65 Tsurumai-cho,

Showa-ku, Nagoya 466-8550, Japan

e-mail: m-kanda@med.nagoya-u.ac.jp related to more advanced GC and subsequent adverse prognosis. Among 72 patients who underwent R0 resection, downregulation of DENND2D mRNA in GC tissues was an independent prognostic factor and associated with early recurrence.

Conclusions Our results suggested that DENND2D is a putative tumor suppressor gene regulated by promoter hypermethylation in GC. Downregulation of DENND2D can serve as a novel tumor biomarker to predict progression and early recurrence of all types of GC.

Keywords Gastric cancer - DENND2D · Expression · Hypermethylation · Prognosis

\section{Introduction}

Gastric cancer (GC) is one of the most common malignant tumors and the second most common cause of cancer death worldwide, although there has been a steady decline in the incidence and mortality risk of GC over several decades in most countries [1, 2]. However, advanced GCs are still likely to have an adverse prognosis as a result of early recurrence and distal metastasis, even after curative resection [3]. Thus, development of novel diagnostic and therapeutic approaches for GC is urgently needed.

$\mathrm{GC}$ is the consequence of a multistep and multifactorial process [4, 5]. Several environmental factors, including Helicobacter pylori infection, consumption of salted and nitrated foods, and cigarette smoking, have been found to be associated with the risk of developing GC [6-9]. In addition to environmental factors, genetic factors also play an important role in GC etiology, and previous reports suggest that combinations of genetic and/or epigenetic changes in oncogenic and tumor suppressor pathways may 
drive GC [10-12]. Based on cancer epidemiology, pathological characteristics, and tumor location, GCs can be categorized into three distinct malignancies arising in the same organ: (1) proximal nondiffuse cancers of the cardia and gastroesophageal junction, which are rapidly increasing in incidence in the Western Hemisphere and are related to lifestyle factors, obesity, and chronic gastric acid reflux; (2) diffuse-type GC derived from poorly differentiated or signet-ring cell adenocarcinoma; and (3) distal nondiffuse intestinal-type GC, linked to precursor lesions arising from atrophic gastritis and chronic inflammation related to Helicobacter pylori [11, 13-15]. Genetic background according to ethnicity may be related to differences in the histological and anatomical subtypes of GC; therefore, analysis taking into account GC subtypes is necessary to clarify molecular mechanisms of GC carcinogenesis and progression.

The DENN/MADD domain-containing (DENND) proteins regulate Rab GTPases and represent a newly recognized class of membrane trafficking proteins [16, 17]. DENND proteins interact directly with Rab35 and function as guanine nucleotide exchange factors (GEF) for this GTPase [17-20]. To date, little is known about the purpose and expression patterns of DENND family proteins in malignancies, although they play important roles in intracellular signaling pathways by integrating the activity of Rab pathways [16, 17]. Membrane trafficking proteins are assumed to have close implications for drug delivery, which is important for GC treatment [21, 22]; however, the role and regulatory mechanisms of DENND family proteins in GC have not been reported. We recently took particular note of DENND2D, which is located on chromosome 1p13.3 and encodes a 53-kDa protein [23], and reported that $D E N N D 2 D$ has tumor suppressive activity in hepatocellular carcinoma [24]. Accordingly, we investigated the expression and regulatory mechanism of $D E N$ $N D 2 D$ to identify a novel biomarker for GC.

\section{Materials and methods}

Sample collection and DNA preparation

Twelve GC cell lines (AZ521, H111, KATOIII, MKN1, MKN28, MKN45, MKN74, NUGC2, NUGC3, NUGC4, SC-2-NU, and SC-6-LCK) were obtained from the American Type Culture Collection (Manassas, VA, USA) or Tohoku University, Japan, and cultured in RPMI-1640 supplemented with $10 \%$ fetal bovine serum in $5 \% \mathrm{CO}_{2}$ at $37{ }^{\circ} \mathrm{C}$. Primary GC tissues and corresponding noncancerous tissues were collected from 112 patients undergoing gastric resection for GC without neoadjuvant therapy at Nagoya University Hospital between 2001 and 2008.
Written informed consent for use of clinical samples and data, as required by the institutional review board at Nagoya University, Japan, was obtained from all enrolled patients. Specimens were classified histologically using the 7 th edition of the Union for International Cancer Control (UICC) classification [25]. Relevant clinicopathological parameters were investigated from medical records. To evaluate whether the expression and methylation status of DENND2D differ by tumor phenotype, patients were categorized into three groups according to the definition of GC subtypes based on tumor location and morphology, as follows: proximal nondiffuse, diffuse, and distal nondiffuse type [5, 14]. Since 2006, adjuvant chemotherapy using S-1 (an oral fluorinated pyrimidine) was applied to all UICC stage II-IV GC patients unless contraindicated by the patient's condition [26, 27].

Collected tissue samples were immediately flash frozen in liquid nitrogen and stored at $-80{ }^{\circ} \mathrm{C}$ until DNA and RNA extraction. Approximately $5 \mathrm{~mm}^{2}$ tumor sample was extracted without necrotic components and confirmed to contain more than $80 \%$ tumor cells [28]. Corresponding noncancerous gastric mucosa samples were obtained from the same patient and were collected $>5 \mathrm{~cm}$ from the edge of the tumors.

Reverse-transcription polymerase chain reaction (RT-PCR) and quantitative real-time RT-PCR (qRT-PCR)

DENND2D mRNA expression levels were analyzed by RT-PCR and qRT-PCR. Total RNA (10 $\mu \mathrm{g}$ per sample) was isolated from 12 GC cell lines and 112 primary GC tissues and corresponding noncancerous tissues and was used to generate complementary DNA. PCR primers and conditions for RT-PCR and qRT-PCR were as described previously [24]. qRT-PCR was performed using $12 \mathrm{GC}$ cell lines and 112 pairs of clinical samples in triplicate and included no-template samples as negative controls. The expression of glyceraldehyde-3-phosphate dehydrogenase $(G A P D H)$ mRNA was quantified in each sample for standardization. The expression level of each sample is shown as the value of $D E N N D 2 D$ divided by that of $G A P D H$. DENND2D mRNA expression was defined as downregulated in tumor tissues when its level was less than half that of the corresponding noncancerous tissues.

Methylation-specific PCR (MSP) and bisulfite sequence analysis

$D E N N D 2 D$ possesses a $\mathrm{CpG}$ island around the promoter region [24], and we hypothesized that aberrant methylation is responsible for regulation of $D E N N D 2 D$ transcription in GC. DNA samples from 12 GC cell lines and 112 GC tissues and corresponding noncancerous tissues were 
treated with bisulfite, and conventional MSP was performed. Genomic bisulfite-treated DNA from GC cell lines was sequenced to ascertain whether the MSP amplification had performed reliably. The primer sequences of MSP and bisulfite sequencing and the PCR conditions were reported previously [24, 29]. In addition, quantitative MSP (QMSP) was conducted to verify accuracy of conventional MSP. Real-time detection for QMSP was done with an ABI StepOnePlus Real-Time PCR System (Perkin-Elmer, Applied Biosystems, Foster City, CA, USA). To quantify the amount of methylated and unmethylated DNA in a DNA sample, a standard curve was generated containing serially diluted bisulfite-modified DNA confirmed to be completely methylated or unmethylated to be described (MKN28 for methylated and NUGC4 for unmethylated) [30]. The DENND2D methylation score was defined as follows: concentration of methylated DNA/(concentration of methylated DNA + unmethylated DNA) [31].

\section{5-Aza-2'-deoxycytidine $(5$-aza-dC) treatment}

To assess the relation of promoter hypermethylation to DENND2D transcription, GC cells $\left(1.5 \times 10^{6}\right)$ were treated with 5-aza-dC (Sigma-Aldrich, St. Louis, MO, USA) to inhibit DNA methylation and cultured for 6 days with medium changes on days 1,3 , and 5. RNA was extracted and RT-PCR was performed as described previously [32].

Immunohistochemistry (IHC)

IHC was conducted to investigate $D E N N D 2 D$ protein localization in 48 representative sections of well-preserved GC tissue. Formalin-fixed, paraffin-embedded tissues were treated with $3 \% \mathrm{H}_{2} \mathrm{O}_{2}$ to inhibit endogenous peroxidase, followed by epitope retrieval via five incubations of $5 \mathrm{~min}$ each in $10 \mathrm{mM}$ citrate buffer at $95^{\circ} \mathrm{C}$. The samples were incubated with Histofine SAB-PO(R) (Nichirei, Tokyo, Japan) for $5 \mathrm{~min}$ to limit nonspecific reactivity and were then incubated for $1 \mathrm{~h}$ at room temperature with a rabbit antibody against DENND2D (HPA043630; Atlas Antibodies, Stockholm, Sweden) diluted 1:25 in Antibody Diluent (Dako). Sections were developed for 5 min using liquid 3,3'-diaminobenzidine as the substrate (Nichirei). Staining patterns were compared between GCs and the corresponding noncancerous tissues. Intensity of DENND2D protein expression was graded depending on the percentage of staining cells as follows: no staining, minimal $(<10 \%)$, focal $(10-50 \%)$, and diffuse $(>50 \%)$ [33, 34]. To avoid subjectivity, specimens were randomized and coded before analysis by two independent observers blinded to the status of the samples. Each observer evaluated all specimens at least twice within a given time interval to minimize intraobserver variation.
Statistical analysis

The relative mRNA expression levels (DENND2D/GAP$D H$ ) between GC and noncancerous tissues were analyzed using the Mann-Whitney $U$ test. The $\chi^{2}$ test was used to analyze the association between the expression and methylation status of $D E N N D 2 D$ and clinicopathological parameters. Disease-specific and disease-free survival rates were calculated using the Kaplan-Meier method, and the difference in survival curves was analyzed using the logrank test. We performed multivariable regression analysis to detect prognostic factors using the Cox proportional hazards model, and variables with a $P$ value $<0.05$ were entered into the final model. All statistical analysis was performed using JMP 10 software (SAS Institute, Cary, NC, USA). $P<0.05$ was considered statistically significant.

\section{Results}

Patient characteristics

Patient age ranged from 21 to 84 years $(63.9 \pm 11.5$ years, mean \pm standard deviation), and the male-to-female ratio was 83:29. Pathologically, 63 patients were diagnosed with undifferentiated GC and 49 with differentiated GC. When categorized into three phenotypes of GC, 24 patients were found to have proximal nondiffuse, 17 diffuse, and 81 distal nondiffuse GC. According to the 7 th edition of the UICC classification, 21, 19, 33, and 39 patients were in stages I, II, III, and IV, respectively, which is to say 72 patients in stages I-III underwent R0 resection. Thirtythree of 39 stage IV patients were diagnosed as stage IV because of positive peritoneal lavage cytology. Six patients in stage IV had synchronous liver metastasis and

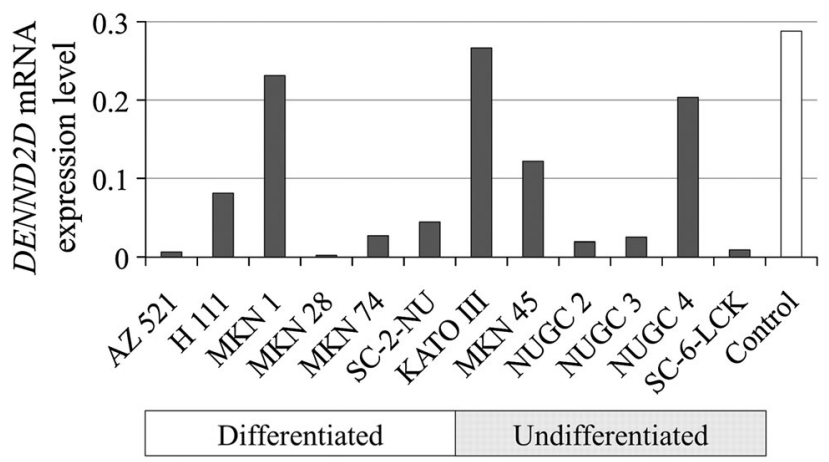

Fig. 1 DENND2D mRNA expression in gastric cancer (GC) cell lines and control samples (median value of 112 noncancerous tissues) by qRT-PCR. Reduced expression was confirmed in all GC cell lines apart from KATO III compared with the control 
Fig. 2 Methylation analysis of $D E N N D 2 D$. a Map overview of the $D E N N D 2 D$ gene. The $\mathrm{CpG}$ island, indicated by the line, was centered on the DENND2D transcription initiation site extending upstream into the promoter region. b Results of conventional methylationspecific (MSP) PCR and quantitative MSP. Complete methylation was observed in AZ521, MKN28, MKN74, and SC-6-LCK cells; partial methylation in H111, MKN45, NUGC2, NUGC3, and SC-2-

$\mathrm{NU}$; and absence of methylation in MKN1, KATO III, and NUGC4 cell lines. c RT-PCR before and after 5-aza-dC treatment. Reactivation of or increases in DENND2D mRNA expression were shown in AZ521, H111, MKN28, MKN45, MKN74 NUGC2, and SC-6-LCK cells. d Bisulfite sequencing analysis. Results of bisulfite sequence analysis in MKN28 and NUGC4 cells shown with MSP. All CpG sites in MKN28 cells were CG (cytosine and guanine); all those of NUGC4 were TG (thymine and guanine) $\mathbf{a}$

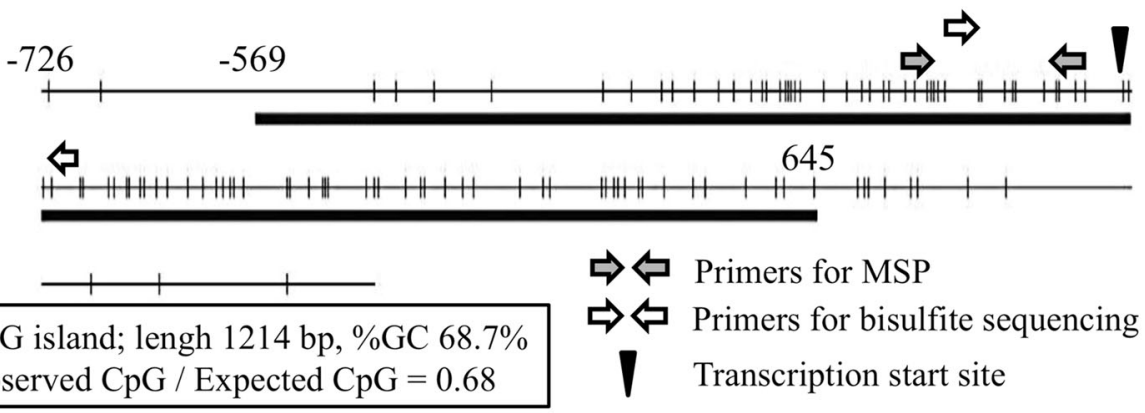

b
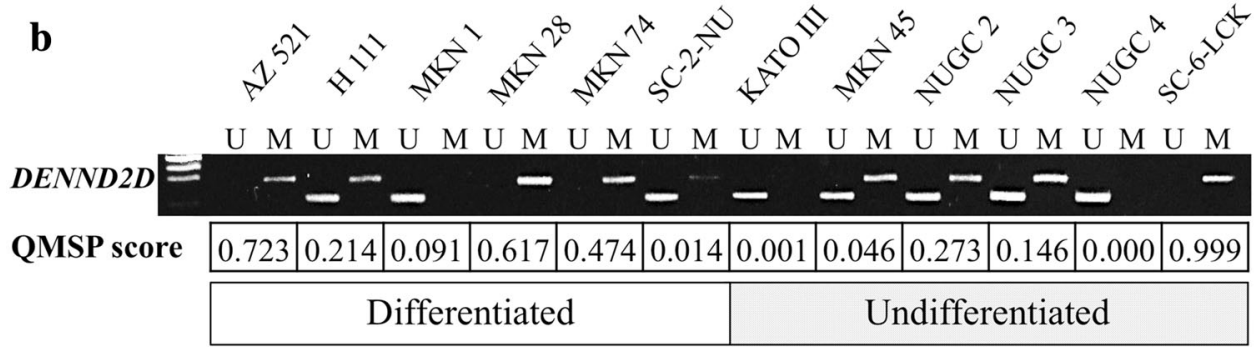

c
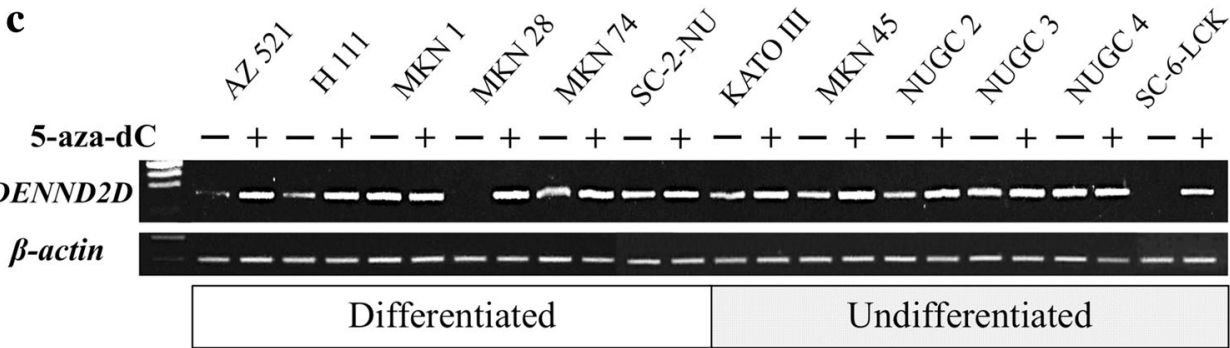

d MKN 28

$\underline{\text { NUGC } 4}$
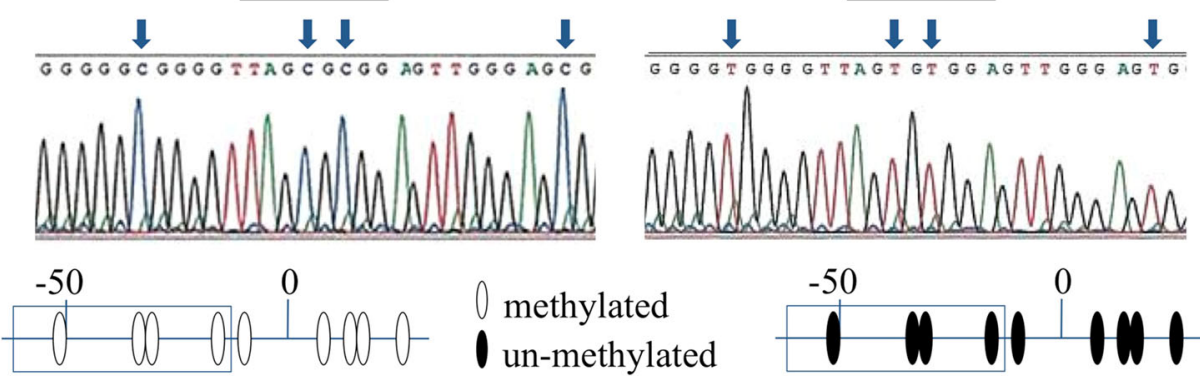

0 methylated
1 un-methylated

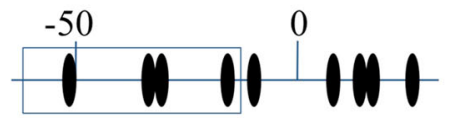

underwent gastrectomy with the purpose of controlling tumor bleeding or obstruction to the passage of food.

\section{DENND2D mRNA expression and methylation analysis} in GC cell lines

Distinct decreases in DENND2D mRNA were confirmed in $9(75 \%)$ of $12 \mathrm{GC}$ cell lines compared with the median expression level in noncancerous gastric tissues. There was no apparent difference in expression pattern between cell lines derived from differentiated and undifferentiated GCs (Fig. 1). In conventional MSP using primers at the promoter region of $D E N N D 2 D$ gene (Fig. 2a), complete methylation occurred in AZ521, MKN28, MKN74, and SC-6-LCK cells and partial methylation in H111, MKN45, NUGC2, NUGC3, and SC-2-NU; there was an absence of methylation in MKN1, KATO III, and NUGC4 cells. QMSP scores were consistent with the results of conventional MSP in all GC cells (Fig. 2b). When comparing the expression of $D E N N D 2 D$ mRNA by semiquantitative RTPCR in GC cell lines before and after treatment with the DNA methylation inhibitor, 5-aza-dC, reactivation or an increase in DENND2D expression was shown in AZ521, H111, MKN28, MKN45, MKN74 NUGC2, and SC-6-LCK cells in conformity with the results of the qPCR and MSP (Fig. 2c). In the direct sequence analysis of MKN28 
(complete methylation in MSP) and NUGC4 (absence of methylation in MSP), we found that all $\mathrm{CpG}$ sites in the MKN28-derived fragment were CG (cytosine and guanine), whereas while all those of NUGC4 were TG (thymine and guanine) (Fig. 2d). These results indicated that the MSP had proceeded correctly.

Expression and methylation status of DENND2D in 112 clinical GC samples

In $89(79 \%)$ of 112 patients, the expression level of $D E N$ $N D 2 D$ mRNA was lower in GC tissues than in the corresponding normal tissues. Frequency of promoter hypermethylation in GC tissues and the corresponding normal tissues was $58 \%$ and $4 \%$, respectively. The GC tissues had a significantly lower expression of DENND2D mRNA and higher prevalence of promoter hypermethylation than the corresponding normal tissues $(P<0.001$ for both; Fig. 3a). The expression levels of DENND2D mRNA in patients with methylated GC were significantly lower than those of the others $(P=0.001)$, indicating promoter hypermethylation contributed to strong suppression of DENND2D transcription. There was no significant difference in DENND2D mRNA expression and methylation status between patients with undifferentiated and differentiated GC (Fig. 3b). When comparing the DEN$N D 2 D$ mRNA expression level and methylation status in GC tissue samples categorized into proximal nondiffuse, diffuse, and distal nondiffuse subtypes, no significant differences were shown among the three groups, suggesting that neither $D E N$ $N D 2 D$ mRNA expression nor methylation status varies by tumor location and morphology (Fig. 3c).

IHC

Representative cases with downregulated DENND2D mRNA expression in GC tissues showed reduced expression of DENND2D protein in the cytoplasm of cancerous components compared with adjacent noncancerous tissue components, corresponding with MSP data (Fig. 4a). Overall, the expression patterns of $D E N N D 2 D$ protein of 48 patients were consistent with the qRT-PCR data. When grading the staining intensity of cancerous components, 4, 16, 19, and 9 patients were categorized in no staining, minimal, focal and diffuse, respectively. The positive correlation between $D E N N D 2 D$ staining grade and mRNA expression level in GC tissues was seen (Fig. 4b).

Prognostic values of expression and methylation status of DENND2D

Forty-nine of 112 GC patients showed substantial downregulation (less than half) of DENND2D mRNA in GC
Fig. 3 Expression and methylation status in clinical tissues. a GC tissues showed lower mean expression of DENND2D mRNA and higher frequency of methylation than corresponding normal tissues. b There were no significant differences in DENND2D mRNA expression level and frequency of methylation in GC tissues between patients with undifferentiated GC and differentiated GC. $N S$, not significant. c DENND2D mRNA expression and methylation frequency in GC tissues were comparable among the three GC subtypes: proximal nondiffuse, diffuse, and distal nondiffuse. NS not significant
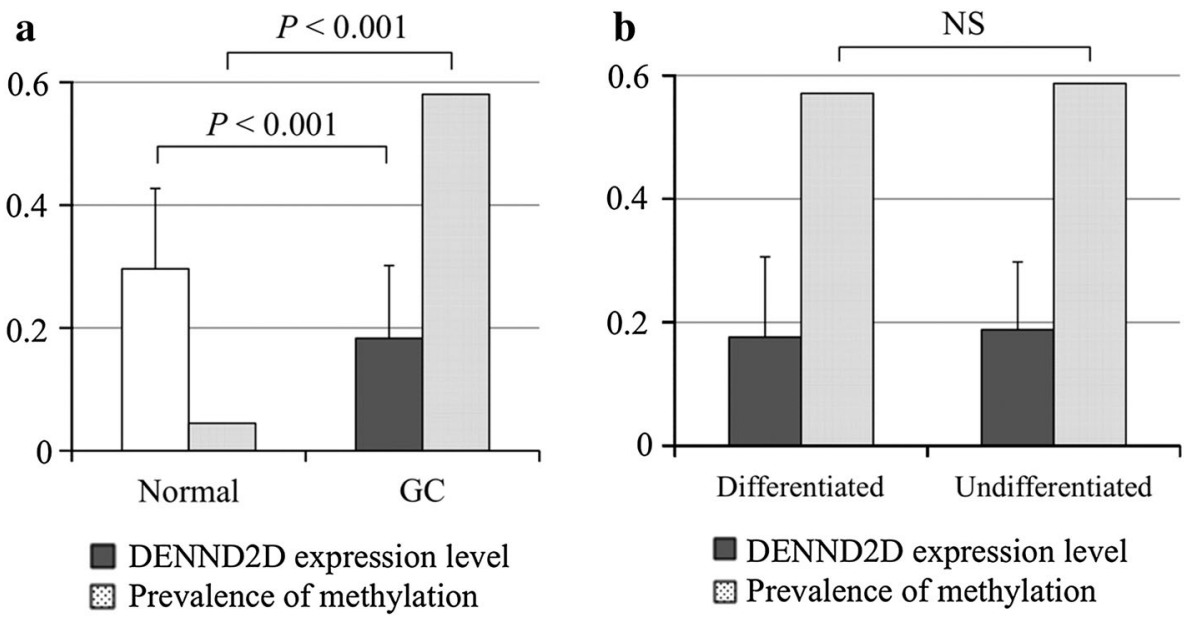

DENND2D expression level 웅 Prevalence of methylation

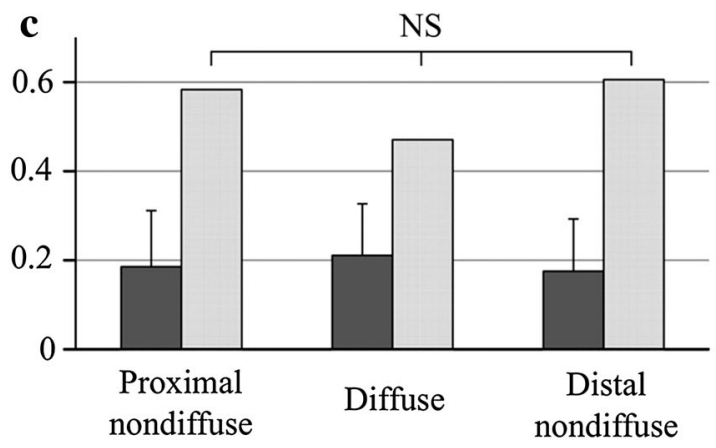

DENND2D

expression level

$\square$ Prevalence of methylation 
Fig. 4 Immunohistochemistry. a Detection of DENND2D protein and MSP in two Patient with diffuse-type poorly differentiated adenocarcinoma perforating serosa (pT4a). $\times 100$. Right: Patient with proximal nondiffuse-type moderately differentiated adenocarcinoma invading submucosa (pT1b). $\times 400$. In both, cancerous tissue components showed reduced DENND2D protein expression compared with adjacent noncancerous tissue components; positive MSP was confirmed in GC tissues.

$N$ noncancerous tissue component, $T$ tumor tissue component. b A positive correlation was seen between expression level of DENND2D mRNA and staining grade in GC tissues representative GC patients. Left:
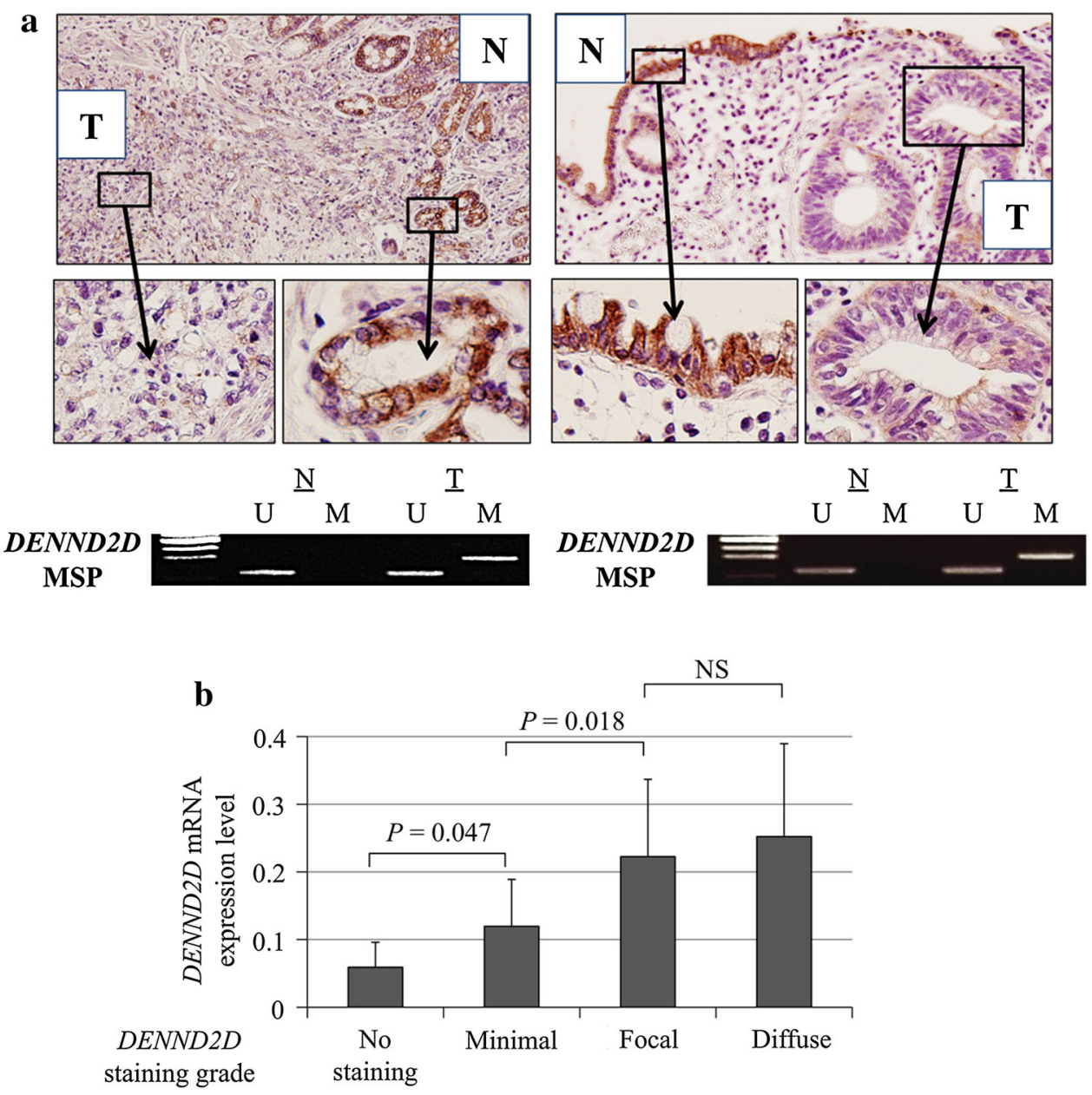

Fig. 5 Prognostic impact of DENND2D mRNA expression in GC patients. a GC patients with downregulated $D E N N D 2 D$ mRNA had significantly shorter disease-specific survival than patients without. b, c Among 72 patients who underwent $\mathrm{R} 0$ resection, patients with downregulated DENND2D mRNA had significantly shorter disease-specific survival (b) and disease-free survival (c) than those who did not. $P$ values were calculated using the logrank test

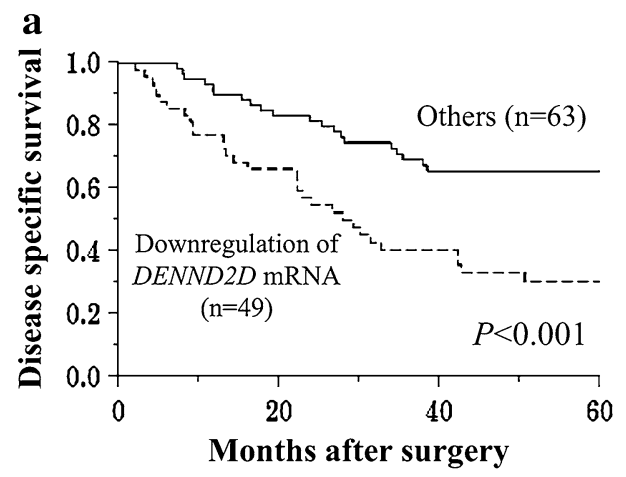

b

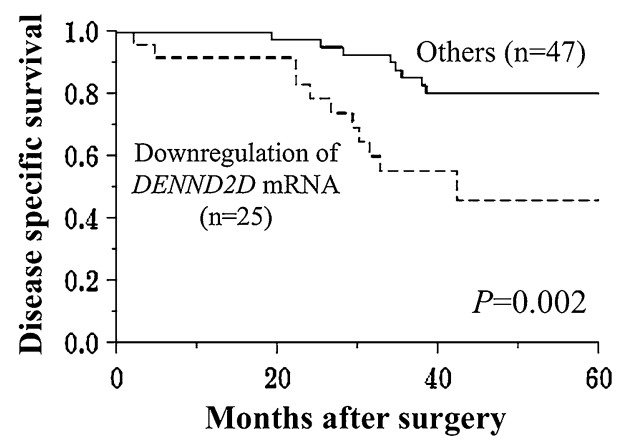

c

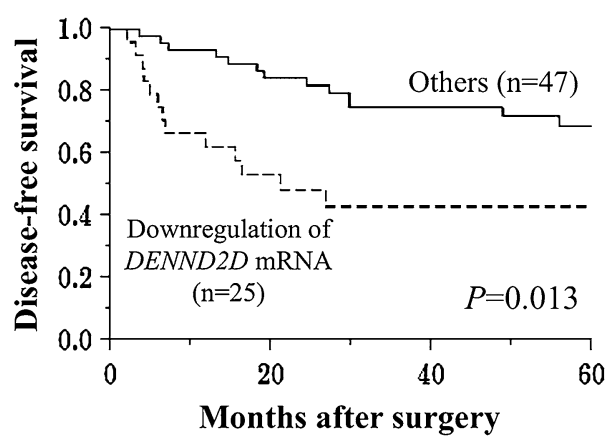


Table 1 Association between expression level of DENND2D mRNA and clinicopathological parameters in 112 gastric cancer (GC) patients

\begin{tabular}{lll}
\hline $\begin{array}{l}\text { Clinicopathological } \\
\text { parameters }\end{array}$ & $\begin{array}{l}\text { Downregulation of } \\
D E N N D 2 D \text { mRNA in GC } \\
\text { tissue }(n)\end{array}$ & $\begin{array}{l}\text { Others } \\
(n)\end{array}$ \\
\end{tabular}

Age

$<65$ years

16

$\geq 65$ years

33

Gender

Male

Female

42

Subtype

Cardiac

Diffuse

Distal

Carcinoembryonic antigen $(\mathrm{ng} / \mathrm{ml})$

$\begin{array}{ll}\leq 5 & 39 \\ >5 & 10\end{array}$

Carbohydrate antigen 19-9 (IU/ml)

$\leq 37$

38

$>37$

11

Tumor size

$<6 \mathrm{~cm}$

19

$\geq 6 \mathrm{~cm}$

30

Tumor depth (UICC)

pT1, pT2 6

pT3, pT4

43

Differentiation

Differentiated 21

Undifferentiated 28

Lymphatic involvement

Absent 5

Present 44

Vessel invasion

$\begin{array}{ll}\text { Absent } & 16 \\ \text { Present } & 33\end{array}$

Lymph node metastasis

$\begin{array}{ll}\text { Absent } & 12 \\ \text { Present } & 37\end{array}$

Present

37

Peritoneal washing cytology

$\begin{array}{llll}\text { Absent } & 34 & 49 & 0.316 \\ \text { Present } & 15 & 14 & \\ \text { UICC stage } & & & \\ \text { I-III } & 24 & 47 & 0.005 \\ \text { IV } & 25 & 16 & \end{array}$

Hypermethylation of DENND2D

\begin{tabular}{llll} 
Absent & 11 & 36 & $<0.001$ \\
Present & 38 & 27 & \\
\hline
\end{tabular}

GC gastric cancer, UICC Union for International Cancer Control tissues compared with noncancerous tissues. Patients with downregulation of DENND2D mRNA expression in GCs had a significantly shorter disease-specific survival than the others (2-year survival rates, $55 \%$ and $82 \%$, respectively, Fig. 5a). Downregulation of DENND2D mRNA in GCs was significantly associated with advanced age, male sex, tumor size $\geq 6 \mathrm{~cm}$, advanced pT stage, vessel invasion, lymph node metastasis, UICC stage IV (presence of distant metastasis), and promoter hypermethylation of DENND2D (Table 1), but not GC subtype.

Next, we performed a subgroup analysis focusing on 72 patients who underwent R0 resection. Patients with downregulated DENND2D mRNA in GCs $(n=25)$ had significantly shorter disease-specific survival than the others $(n=47)$ (2-year survival rates: $79 \%$ and $95 \%$, respectively, $P=0.002$; Fig. $5 b$ ). Univariate analysis for disease-specific survival showed that carbohydrate antigen 19-9 of $>37 \mathrm{IU} / \mathrm{ml}$, pT4, lymphatic involvement, lymph node metastasis, hypermethylation of DENND2D, and downregulation of $D E N N D 2 D$ mRNA in GC tissues were significantly prognostic of adverse outcomes. Multivariate analysis identified downregulation of DENND2D mRNA as an independent prognostic factor (hazard ratio 4.04, $95 \%$ confidence interval, $1.29-13.7, P=0.016$; Table 2). In terms of disease-free survival, patients with substantial downregulation of DENND2D mRNA in GCs had significantly earlier recurrences after surgery than the others (2year disease-free survival rates: $48 \%$ and $82 \%$, respectively, $P=0.013$; Fig. 5c).

\section{Discussion}

Because membrane trafficking proteins have intimate involvement in the development, progression, and drug sensitivity property of cancers, the functions of Rab GTPases in malignancies have been investigated in recent years [21, 22, 35]. Chemotherapy is a key therapeutic strategy for advanced GC [5, 27]; therefore, exploration of the role of the DENND family of membrane trafficking proteins is essential for further understanding of the underlying molecular mechanisms in GC.

In this study, $D E N N D 2 D$ was identified as a putative tumor suppressor gene (TSG) inactivated epigenetically in GC. We have shown here that the level of DENND2D mRNA expression was reduced in the majority of GC cell lines and surgical specimens accompanying promoter hypermethylation, and the mean expression level was significantly lower in GC tissues than in corresponding normal tissues. This result indicates that $D E N N D 2 D$ may play an important role in gastric carcinogenesis. The expression pattern of $D E N N D 2 D$ protein using IHC was confirmed to 
Table 2 Prognostic factors for disease-specific survival in 72 GC patients who underwent R0 resection

\begin{tabular}{|c|c|c|c|c|c|c|c|}
\hline \multirow[t]{2}{*}{ Variable } & \multirow[t]{2}{*}{$n$} & \multicolumn{3}{|l|}{ Univariate } & \multicolumn{3}{|l|}{ Multivariate } \\
\hline & & Hazard ratio & $95 \% \mathrm{CI}$ & $P$ value & Hazard ratio & $95 \% \mathrm{CI}$ & $P$ value \\
\hline Age ( $\geq 65$ years $)$ & 37 & 1.41 & $0.58-3.61$ & 0.444 & & & \\
\hline Gender (male) & 55 & 1.80 & $0.61-7.73$ & 0.314 & & & \\
\hline Subtype (distal) & 50 & 0.50 & $0.21-1.25$ & 0.136 & & & \\
\hline Carcinoembryonic antigen $(>5 \mathrm{ng} / \mathrm{ml})$ & 10 & 1.76 & $0.50-4.82$ & 0.340 & & & \\
\hline Carbohydrate antigen 19-9 (>37 IU/ml) & 11 & 3.82 & $1.34-9.63$ & 0.014 & 3.83 & $1.10-12.9$ & 0.035 \\
\hline Tumor length $(\geq 60 \mathrm{~mm})$ & 28 & 1.92 & $0.80-4.78$ & 0.145 & & & \\
\hline Tumor depth (pT4, UICC) & 25 & 2.45 & $1.01-6.08$ & 0.047 & 2.61 & $1.03-6.79$ & 0.044 \\
\hline Tumor differentiation (undifferentiated) & 35 & 1.97 & $0.81-5.25$ & 0.138 & & & \\
\hline Lymphatic involvement & 56 & 6.33 & $1.31-114$ & 0.017 & 2.33 & $0.32-47.6$ & 0.437 \\
\hline Vessel invasion & 32 & 1.95 & $0.80-4.97$ & 0.140 & & & \\
\hline Lymph node metastasis & 36 & 7.12 & $2.39-30.5$ & $<0.001$ & 2.91 & $0.78-15.8$ & 0.119 \\
\hline Hypermethylation of $D E N N D 2 D$ & 41 & 3.68 & $1.35-12.8$ & 0.010 & 2.42 & $0.80-9.15$ & 0.123 \\
\hline Downregulation of $D E N N D 2 D$ mRNA & 25 & 3.71 & $1.53-9.48$ & 0.004 & 4.04 & $1.29-13.7$ & 0.016 \\
\hline
\end{tabular}

$G C$ gastric cancer, $C I$ confidence interval, UICC Union for International Cancer Control

be consistent with that of its mRNA levels, as analyzed by qPCR. Moreover, a significant reduction in DENND2D mRNA levels in GC tissues was associated with factors related to more advanced GC, including distant metastasis and subsequent adverse prognosis, suggesting that $D E N$ $N D 2 D$ acts as a TSG mediated through promoter hypermethylation, consistent with the results of previous studies of $D E N N D 2 D$ expression in lung cancer and hepatocellular carcinoma [23, 24]. Reactivation of DENN2D mRNA expression after treatment with 5-aza-dC, a DNA methyltransferase (DNMT) inhibitor, indicated that $D N M T$ was thought to have a role in $D E N N D 2 D$ transcription [36]. Accordingly, DNMT suppression may be a key to activate the tumor-suppressive function of DENND2D.

Another striking finding was that the substantial downregulation of $D E N N N 2 D$ mRNA was an independent prognostic factor and associated with early recurrence after R0 resection, implying $D E N N D 2 D$ expression could be used as a prognostic marker for early recurrence and progression of GC. Interestingly, expression patterns and methylation of $D E N N D 2 D$ did not differ among the three GC subtypes examined. DENND2D was found to act consistently in all GC types.

Each $D E N N D 2$ protein acts as a GEF for Rab9a/b. Rab GTPases regulate tumorigenesis via trafficking-mediated events, and some function in a trafficking-independent manner [22, 37-40]. Additionally, it is known that several members of the Rab GTPase family affect exosome secretion via the trans-Golgi network or by inducible vesicular trafficking [35, 41]. Exosomes released from cancer cells can be taken up by neighboring cells and are capable of inducing pathways involved in cancer initiation and progression [42, 43]. Therefore, it is thought that
$D E N N D 2 D$ plays an important role in carcinogenesis and cancer progression via regulation of Rab GTPases. We performed a cell proliferation assay using water-soluble tetrazolium salts 1 (WST-1) to evaluate the function of DENND2D; however, no significant correlations between DENND2D mRNA expression level and the proliferative activity of GC cells were observed (data not shown). This result implied that downregulation of DENND2D might promote GC progression through multiple functions. When considering the next step, investigation of the relationship between DENND2D expression, mucin phenotype, and chemoresistance could be of great help in further clarifying the role played by $D E N N D 2 D$ in carcinogenesis and progression of GC.

In conclusion, $D E N N D 2 D$ acts as a putative TSG that is inactivated by promoter hypermethylation in GC and can serve as a promising biomarker of recurrence and progression in any type of GC.

Conflict of interest None declared.

\section{References}

1. Parkin DM, Bray F, Ferlay J, Pisani P. Global cancer statistics, 2002. CA Cancer J Clin. 2005;55:74-108.

2. Jang BG, Kim WH. Molecular pathology of gastric carcinoma. Pathobiology. 2011;78:302-10.

3. Wagner AD, Grothe W, Haerting J, Kleber G, Grothey A, Fleig WE. Chemotherapy in advanced gastric cancer: a systematic review and meta-analysis based on aggregate data. J Clin Oncol. 2006;24:2903-9.

4. Gonzalez CA, Agudo A. Carcinogenesis, prevention and early detection of gastric cancer: where we are and where we should go. Int J Cancer. 2012;130:745-53. 
5. Hartgrink HH, Jansen EP, van Grieken NC, van de Velde CJ. Gastric cancer. Lancet. 2009;374:477-90.

6. Larsson SC, Orsini N, Wolk A. Processed meat consumption and stomach cancer risk: a meta-analysis. J Natl Cancer Inst. 2006;98:1078-87.

7. Shikata K, Doi Y, Yonemoto K, Arima H, Ninomiya T, Kubo M, et al. Population-based prospective study of the combined influence of cigarette smoking and Helicobacter pylori infection on gastric cancer incidence: the Hisayama Study. Am J Epidemiol. 2008;168:1409-15.

8. Tramacere I, Negri E, Pelucchi C, Bagnardi V, Rota M, Scotti L, et al. A meta-analysis on alcohol drinking and gastric cancer risk. Ann Oncol. 2012;23:28-36.

9. Vogiatzi P, Vindigni C, Roviello F, Renieri A, Giordano A. Deciphering the underlying genetic and epigenetic events leading to gastric carcinogenesis. J Cell Physiol. 2007;211:287-95.

10. El-Omar EM, Carrington M, Chow WH, McColl KE, Bream JH, Young HA, et al. Interleukin-1 polymorphisms associated with increased risk of gastric cancer. Nature (Lond). 2000;404: 398-402.

11. Janjigian YY, Kelsen DP. Genomic dysregulation in gastric tumors. J Surg Oncol. 2013;107:237-42.

12. Yasui W, Sentani K, Sakamoto N, Anami K, Naito Y, Oue N. Molecular pathology of gastric cancer: research and practice. Pathol Res Pract. 2011;207:608-12.

13. Lauren $P$. The two histological main types of gastric carcinoma: diffuse and so-called intestinal-type carcinoma. An attempt at a histo-clinical classification. Acta Pathol Microbiol Scand. 1965;64:31-49.

14. Shah MA, Khanin R, Tang L, Janjigian YY, Klimstra DS, Gerdes $\mathrm{H}$, et al. Molecular classification of gastric cancer: a new paradigm. Clin Cancer Res. 2011;17:2693-701.

15. Steevens J, Botterweck AA, Dirx MJ, van den Brandt PA, Schouten LJ. Trends in incidence of oesophageal and stomach cancer subtypes in Europe. Eur $\mathbf{J}$ Gastroenterol Hepatol. 2010;22:669-78.

16. Imai A, Ishida M, Fukuda M, Nashida T, Shimomura H. MADD/ DENN/Rab3GEP functions as a guanine nucleotide exchange factor for Rab27 during granule exocytosis of rat parotid acinar cells. Arch Biochem Biophys. 2013;536:31-7.

17. Marat AL, Dokainish H, McPherson PS. DENN domain proteins: regulators of Rab GTPases. J Biol Chem. 2011;286:13791-800.

18. Marat AL, McPherson PS. The connecdenn family, Rab35 guanine nucleotide exchange factors interfacing with the clathrin machinery. J Biol Chem. 2010;285:10627-37.

19. Stenmark H. Rab GTPases as coordinators of vesicle traffic. Nat Rev Mol Cell Biol. 2009;10:513-25.

20. Yoshimura S, Gerondopoulos A, Linford A, Rigden DJ, Barr FA. Family-wide characterization of the DENN domain Rab GDPGTP exchange factors. J Cell Biol. 2010;191:367-81.

21. Subramani D, Alahari SK. Integrin-mediated function of Rab GTPases in cancer progression. Mol Cancer. 2010;9:312.

22. Cheng KW, Lahad JP, Gray JW, Mills GB. Emerging role of RAB GTPases in cancer and human disease. Cancer Res. 2005;65:2516-9.

23. Ling B, Zheng H, Fu G, Yuan J, Shi T, Chen S, et al. Suppression of non-small cell lung cancer proliferation and tumorigenicity by DENND2D. Lung Cancer. 2013;79:104-10.

24. Kanda M, Nomoto S, Oya H, Takami H, Hibino S, Hishida M, et al. Downregulation of DENND2D by promoter hypermethylation is associated with early recurrence of hepatocellular carcinoma. Int J Oncol. 2014;44:44-52.

25. International Union Against Cancer. TNM classification of malignant tumors. 7th ed. New York: Wiley-Blackwell; 2009.

26. Sakuramoto S, Sasako M, Yamaguchi T, Kinoshita T, Fujii M, Nashimoto A, et al. Adjuvant chemotherapy for gastric cancer with S-1, an oral fluoropyrimidine. $\mathrm{N}$ Engl $\mathrm{J}$ Med. 2007;357:1810-20.

27. Sasako M, Sakuramoto S, Katai H, Kinoshita T, Furukawa H, Yamaguchi T, et al. Five-year outcomes of a randomized phase III trial comparing adjuvant chemotherapy with S-1 versus surgery alone in stage II or III gastric cancer. J Clin Oncol. 2011;29:4387-93.

28. Kanda M, Nomoto S, Nishikawa Y, Sugimoto H, Kanazumi N, Takeda $\mathrm{S}$, et al. Correlations of the expression of vascular endothelial growth factor B and its isoforms in hepatocellular carcinoma with clinico-pathological parameters. J Surg Oncol. 2008;98:190-6.

29. Kanda M, Nomoto S, Okamura Y, Nishikawa Y, Sugimoto H, Kanazumi N, et al. Detection of metallothionein $1 \mathrm{G}$ as a methylated tumor suppressor gene in human hepatocellular carcinoma using a novel method of double combination array analysis. Int $\mathbf{J}$ Oncol. 2009;35:477-83.

30. Matsubayashi H, Canto M, Sato N, Klein A, Abe T, Yamashita K, et al. DNA methylation alterations in the pancreatic juice of patients with suspected pancreatic disease. Cancer Res. 2006;66:1208-17.

31. Nakayama G, Hibi K, Kodera Y, Kodera Y, Koike M, Fujiwara M, Nakao A. P16 methylation in serum as a potential marker for the malignancy of colorectal carcinoma. Anticancer Res. 2007;27:3367-70.

32. Kanda M, Nomoto S, Okamura Y, Hayashi M, Hishida M, Fujii $\mathrm{T}$, et al. Promoter hypermethylation of fibulin 1 gene is associated with tumor progression in hepatocellular carcinoma. Mol Carcinog. 2011;50:571-9.

33. Solez K, Colvin RB, Racusen LC, Haas M, Sis B, Mengel M, et al. Banff 07 classification of renal allograft pathology: updates and future directions. Am J Transplant. 2008;8:753-60.

34. Loupy A, Hill GS, Suberbielle C, Charron D, Anglicheau D, Zuber J, et al. Significance of C4d Banff scores in early protocol biopsies of kidney transplant recipients with preformed donorspecific antibodies (DSA). Am J Transplant. 2011;11:56-65.

35. Ostrowski M, Carmo NB, Krumeich S, Fanget I, Raposo G, Savina $\mathrm{A}$, et al. Rab27a and Rab27b control different steps of the exosome secretion pathway. Nat Cell Biol. 2010;12:19-30.

36. Tsai CT, Yang PM, Chern TR, Chuang SH, Lin JH, Klemm L, et al. AID downregulation is a novel function of the DNMT inhibitor 5-aza-deoxycytidine. Oncotarget. 2013. Nov 25. Epub ahead of print.

37. Bloethner S, Mould A, Stark M, Hayward NK. Identification of ARHGEF17, DENND2D, FGFR3, and RB1 mutations in melanoma by inhibition of nonsense-mediated mRNA decay. Genes Chromosomes Cancer. 2008;47:1076-85.

38. Croizet-Berger K, Daumerie C, Couvreur M, Courtoy PJ, van den Hove MF. The endocytic catalysts, Rab5a and Rab7, are tandem regulators of thyroid hormone production. Proc Natl Acad Sci USA. 2002;99:8277-82.

39. Pawson T, Nash P. Assembly of cell regulatory systems through protein interaction domains. Science. 2003;300:445-52.

40. Sato M, Sato K, Liou W, Harada A, Grant BD. Regulation of endocytic recycling by $C$. elegans Rab35 and its regulator RME4, a coated-pit protein. EMBO J. 2008;27:1183-96.

41. Ponnambalam S, Baldwin SA. Constitutive protein secretion from the trans-Golgi network to the plasma membrane. Mol Membr Biol. 2003;20:129-39.

42. Henderson MC, Azorsa DO. The genomic and proteomic content of cancer cell-derived exosomes. Front Oncol. 2012;2:38.

43. Rink J, Ghigo E, Kalaidzidis Y, Zerial M. Rab conversion as a mechanism of progression from early to late endosomes. Cell. 2005;122:735-49. 